\title{
In Silico Identification of Potential Therapeutic Agents for COVID-19 Based on Molecular Docking Study of Main Protease and Receptor Binding Domain of Spike Protein.
}

\author{
Vajiheh Eskandari ( $\nabla$ veskandari@znu.ac.ir) \\ University of Zanjan https://orcid.org/0000-0002-4639-504X
}

\section{Research Article}

Keywords: Coronavirus, Receptor binding domain, Main-protease, Drug discovery, Docking and Molecular dynamics.

Posted Date: June 28th, 2021

DOI: https://doi.org/10.21203/rs.3.rs-285026/v1

License: (9) This work is licensed under a Creative Commons Attribution 4.0 International License. Read Full License 


\section{Abstract}

Severe acute respiratory syndrome coronavirus (SARS-CoV-2) enter the cell by interacting with human angiotensin-converting enzyme 2 (ACE2) receptor through the receptor-binding domain (RBD) of Sprotein. In the cell the viral 3-chymotrypsin-like cysteine protease (3CLp) enzyme is essential for its life cycle and controls coronavirus replication. Therefore the S-RBD and 3CLp are hot targets for drugs discovery against SARS-CoV-2. This study was to identify repurposing drugs using in-silico screening, docking and molecular dynamics simulation. The study identified Dibenzoyl Thiamine, Folic Acid and Vitamin B12 against the RBD of S-protein and Dibenzoyl Thiamine, Folic Acid, Fursultiamine and Riboflavin to $3 \mathrm{CLp}$. The strong and stable binding of these safe and cheap vitamins at the important residues (R403, K417, Y449, Y453, N501 and Y505) in S-protein -ACE2 interface and 3CLp active site residues (His 41 and Cys 145), indicating that they could be valuable repurpose drugs for inhibiting SARSCoV-2 entry into the host and replication.

\section{Introduction}

The ongoing covid-19 pandemic has resulted in over 71,051,805 affected individuals and 1,608,648 deaths by the end of 14 December 2020 (World Health Organization) [1]. Therefore, it is vital to control and prevention this spreadable disease.

Coronaviruses (CoVs) belong to group of positive single-stranded (+ ss) RNA viruses that classified in the family Coronaviridae [2]. The coronaviradae family includes four genera ( $a, \beta, \gamma$, and $\delta)$, are enveloped viruses [2-3]. The SARS-COV-2 genome comprises approximately $30 \mathrm{~kb}$ nucleotides, which contains 10 Open Reading Frames (ORFs). The $5^{\prime}$ terminal region contains two large replicase ORFs, ORF1a and ORF1b, which encodes two viral polyproteins, pp1a and pp1ab. The autocatalytically processing of polyproteins pp1a and pp1ab, results in production of 16 non-structural proteins (nsp1 to nsp16) (Fig. 1A). Among them, nsp5 (3CLpro enzyme) is indispensable to the viral replication and infection process, therefore is considered as an interesting target for the development of potential inhibitors against covid-19 [4-6].

The 3-D structure of 3CLpro enzyme, also called Main protease (Mpro), contains three domains. Domain I (residues 1-100) and domain II (residues 101-183) have a two- $\beta$-barrel fold, which is alike to chymotrypsin, whereas the third domain (residues 198-303) consists of five a-helical structures. The substrate binding cleft, located between domain I and II, with a catalytic dyad of His41 and Cys145, in which the cysteine thiol allow the nucleophilic attacks and His acts as a proton acceptor (Fig. 1B). Previous studies have shown other residues Thr25, Met49, Phe140. Gly143, His163, Met165, Glu166, His172 and Gln189, which can also interact with ligands [6-7].

The 3 ' terminal of a coronavirus genome encodes structural viral proteins: nucleocapsid $(\mathrm{N})$ protein, membrane (M) protein, envelope (E) protein and spike (S) protein (Fig. 1C). The spike protein localizes on the virion surface and consists of ectodomain region (ED), intracellular domain and TM region [8]. The ED 
region (S1 + S2) (aa 13-1273) includes S1 subunit (aa 13-685) mediating SARS-CoV attachment to host angiotensin-converting enzyme 2 receptor via receptor-binding domain (aa 319-541) and S2 chain (aa 686-1273), which serving host and viral membrane fusion as a result, potentiate the CoV to release its RNA genome in host cell. [8-9]. Several studies have recently, clarifying the residues are involved in interaction between RBD and human ACE2. The key residues of Spike RBD interact with ACE2 receptor are as follow: Arg403, Glu406, Gly446, Lys417, Tyr449, Tyr453, Ala475, Asn487, Gln493, Tyr495, Gly496, Phe497, Gln498, Thr500, Asn501 and Tyr505 [10-11] Among the above residues Lys 417, Tyr 449, Tyr 489, Gln 493, Asn 501 and Tyr 505 play crucial role in anchoring RBD to ACE2 receptor (Fig. 1B) [11]. It therefore appears designing drugs for inhibiting the interaction of the S-protein with its receptor, to be also another attractive strategies for controlling of SARS-cov-2

In this study to find the potent drug molecules to inhibit main protease and also prevent the spike protein interaction with the host receptor, virtual screening was performed against vitamins library in Selleckchem Inc (WA, USA) and subsequently molecular dynamics simulation carried out on selected ligands. Dibenzoyl Thiamine, Folic Acid, Riboflavin, Fursultiamine, Benfotiamine, Calcipotriene, Cocarboxylase and Ergosterol are discovered as potent inhibitor against 3CLp and Vitamin B12 and Dibenzoyl Thiamine, Folic acid and Benfotiamine were found to inhibit RBD of spike protein.

\section{Methods}

Receptors and Ligands preparation:

The crystal structures of receptor-binding domain/RBD of the spike protein (PDB ID.6M0J_E) and 3CLprotease (PDB ID. 6LU7_A) were obtained from the Protein Data Bank[12] and after a cleaning with Discovery Studio 4.1[13], minimized and changed to pdbqt format using AutoDockTools 1.5.6 [14].

For docking against RBD of the spike protein and 3CL-protease, 47 FDA approved vitamins were collected from Selleckchem Inc and the 3D structure of the ligands were retrieved from PubChem database [15] in SDF file format and converted to pdb and pdbqt format using Avogadro[16] and AutoDockTools-1.5.6, resprctively,

\section{Molecular Docking And Post Docking Analysis}

To compute binding affinity between protein and ligands, AutoDock vina in PyRx 0.8 [17] was used to perform the docking based virtual screening over 47 candidate compounds against the 3-D structure of 6lu7 amd 6M0J proteins. For 3CLp inhibition calculation, the grid box was set at $26 A^{\circ} \times 26 A^{\circ} \times 26 A^{\circ}(x$, $y$ and $z)$ and center $-15.518 A^{\circ} \times 21.151 A^{\circ} \times 66.865 A^{\circ}(x, y$ and $z)$ with grid point spacing of one Angstrom. The grid box was set into the His41, Cys145 (catalytic dyad) at ligand binding site.

For the RBD domain of S-protein inhibition calculation, the default parameters for grid box were set to 36 $A^{\circ} \times 55 A^{\circ} \times 32 A^{\circ}(x, y$ and $z)$ and center $-33.688 A^{\circ} \times 30.917 A^{\circ} \times 7.143 A^{\circ}(x, y$ and $z)$ to cover the 
possible binding sites of the SARS-CoV-2 S-protein with the ACE-2 human receptor, in agreement with previous results [11].

The ligands with the highest affinity binding scores -i.e., the most negative binding energies- were selected for more analysis. The protein-ligand complexes were visualized by Discovery Studio 4.1. The hydrophobic interactions and hydrogen bonds of the selected docked complexes were analyzed by Discovery Studio and LigPlot+ (v 1.4.5) [18].

\section{Molecular Dynamic Simulation}

In order to assess the stability of the protein-ligands complexes, molecular dynamics (MD) simulation was performed on docked complexes using GROMACS package 2019.6 [19]. Amber99SB-ILDN [20] forcefield was applied for simulations. The aqueous environment were created using simple point charge TIP3P water model in a cubic box with a distance of $0.8 \mathrm{~nm}$ from the box to the surface of the protein. Steepest descent algorithm was utilized for energy minimization. For each simulation, 50000 steps of energy minimization were performed. The systems were equilibrated using NVT and NPT ensembles for $200 \mathrm{ps}$. The pressure and temperature were set to 1 bar and $300 \mathrm{~K}$. Finally the $100 \mathrm{~ns}$ MD simulation production runs were carried out on ligand-receptor complexes. All simulations were repeated three times. GROMACS in-built tools were applied to analyzing the MD trajectories to calculate the root-mean-square deviation (RMSD), root-mean-square fluctuation (RMSF) and, Xmgrace was used for plotting graphs. Molecular dynamic was done on dimer form of 6lu7, which was retrieved using PyMOL [21].

In another attempt for comparing the variation of RMSD, the RMSD standard deviation (RMSD-SD) was calculated for each residue through MD simulation among the three repeated MD trajectories.

\section{Result And Discussion}

\section{Virtual screening of vitamins against the key viral proteins and analysis.}

Many of the current researches focused on repurposing FDA approved drugs for anti-SARS drug development. In this work 47 approved FDA vitamins were virtual docked against $3 \mathrm{CL}$ - protease and SRBD of coronavirus2.

As shown in Table 1, the binding energy of Folic Acid (vitamin B9 or vitamin M), Dibenzoyl Thiamin (Bentiamin), Riboflavin (vitamin B2), Fursultiamine, Ergosterol, Calcipotriene (Calcipotrio), Cocarboxylase (Thiamine pyrophosphate hydrochloride) and Benfotiamine to 3CLpro (PDBid: 6lu7) exhibit the appropriate score in comparison to indinavir (as docking control), which is HIV protease inhibitor.

For the S-RBD, the Vitamin B12, Folic Acid, Dibenzoyl Thiamine and Benfotiamine displayed the highest affinity (Table 1 ) and good characterization results. 
The selected compounds were visually inspected using Discovery Studio to determine their residues which involved in interactions with 3CLpro and S-RBD. The results in Table 1 show that all selected ligands interact with the catalytic dyad residues (Cys-145 and His-41) as well as significant interactions with the most ligand -binding residues, id; Thr24, Thr25, Thr26, Met49, Asn142, Gly143, His164, Glu166 and $\mathrm{G} \ln 189$ in the binding pocket of $3 \mathrm{Clp}$.

For spike protein, the ligands covers the major part of the RBD-ACE2 interface and interact with important residues in RBD-ACE-2 interface, such as: R403, K417, Y449, Y453, S494, N501 and Y505. Although the 487 and 475 don't fall into traps, but the cover is created over S- RDB seems a barrier for interaction between Spike RBD and human ACE2 2 interface (Table 1).

\section{Md Simulations And Ligplot Analysis}

RMSD analysis of the protein evaluate its conformational and examines whether simulation has equilibrated.

The RMSD plots shown in Figs. 2A and 2B, demonstrated 6lu7-ligand complex structures, except 6lu7ligand 2 , reached a steady state during first few nanoseconds and remained stable throughout the simulation. For 6lu7-ligand 2 very mild variation observed during simulation. This slight instability may be due to the elongated structure of ligand 2, which couldn't not fits well into the 6lu7' binding pocket.

For $6 \mathrm{~m} 0$ j-ligand complexes, due to the special structure of it which doesn't have specific binding pocket, shows a variety of behaviors with different ligands. In relation to $6 \mathrm{~m} 0$ j-ligand 1 , a relatively sharp deviation was seen about 20 nanoseconds for two repeats, and then the system reached equilibrium. (Fig. 2B). The simulation of $6 \mathrm{m0j-ligand} 2$ also showed deviation for three repeats, which could be explained by the special properties of the substrate and the ligand. $6 \mathrm{~m} 0$ j-ligand 5 exhibits a steady increase during the first $20 \mathrm{~ns}$, and reached a stable state throughout equilibration. The $6 \mathrm{m0j}$-vitamin B12 (ligand6) complex presented an acceptable equilibration during simulation.

Comparison of the standard deviation of backbone RMSD among the three repeated simulation of all protein-ligand complexes revealed small SD values $\left(<1 A^{\circ}\right)$, which indicated all simulated protein-ligand complexes are stable (Fig. 2A-B).

RMSF measures the fluctuations of each residue during simulation. The RMSF plot for each residue of the 6lu7-ligand complexes indicates that the protein was not fluctuated in the $100 \mathrm{~ns}$ simulation periods (Fig. 2C). Comparatively, the RMSF plot of the $6 \mathrm{m0j}$ complexes (Fig. 2D) demonstrating overall stability of these constructs, with the exception of a few residues in C-terminal domains that show greater fluctuations. Investigation in $6 \mathrm{~m} 0 \mathrm{j} 3 \mathrm{D}$-structure indicated that residues in loop region are more fluctuated during the simulation. This indicates that the complexes were not fluctuated in the simulation periods.

The top 10 compounds including the compounds selected for the MD study, were subjected to ligplot analysis. 
Investigation in the LigPlot of the 6lu7-Dibenzoyl Thiamine, 6lu7-Fursultiamine, 6lu7-Folic Acid and 6lu7Riboflavin shows that the catalytic dyad residues of both chains of dimer molecule bind to Folic Acid and Dibenzoyl Thiamine ligands. These results are comparable and consistent with the results of LigPlot before MD and Docking results (Fig. 3, Table 1 and Supplementary Fig. 1).

Examination and comparison of the two-dimensional representation of $6 \mathrm{moj}$ interactions with selective ligands after and before MD show the compatibility of the amino acids involved in the interaction with the residues present in the 6moj-S-RBD interface (Fig. 3, Table 1).

These results indicate the stability of the interaction between the selected ligands with the respective receptors.

The above results suggest that vitamins compounds might be the potential 3CLpro and S-RBD inhibitors and could probably be used for treating SARS-CoV-2.

The description of selected compounds are as below:

Benfotiamine is a thioester that is a synthetic S-acyl derivative of thiamine (vitamin B1). Benfotiamine may also be beneficial for the treatment and preventing diabetic nephropathy and type 2 diabetes mellitus [15-Selleckchem].

Cocarboxylase is a thiamine (vitamin B1) derivative which is produced by the enzyme thiamine diphosphokinase (Selleckchem). Thiamine pyrophosphate were found less than toxic in comparison to thiamine and thiamine monophosphate. Thiamine pyrophosphate chloride used in food supplements, as sources of vitamin B1 [25].

Calcipotriene (Calcipotriol, MC903) is a synthetic derivative of calcitriol, a form of vitamin D (Selleckchem). Calcipotriene is used as cream or solution drug in controlling of in psoriasis, and lead to normalization of epidermal growth [27]. The side effects of this drug is very limited [28].

Dibenzoyl Thiamine, a lipophilic derivative of vitamin B (thiamine), is a kind of food additive that can be rapidly absorbed into the body and converted to thiamine (Selleckchem). It appears to break down incompletely to thiamine [22], therefore there will always be some of it in our body intact and may be able to inhibit 3Clp. Bentiamine has been approved as a food additive in Japan [22]. The toxicity, teratogenicity and mutagenicity studies have been showed no side effects for Bentiamine [23].

Folic Acid, a B vitamin, plays an important role in cell division and in the synthesis of amino acids and nucleic acids like DNA (Selleckchem). Currently, this vitamin is prescribed as an oral supplement for all women planning, or capable of, pregnancy. Also, due to the function of folate for detoxification of arsenic through arsenic methylation, folic acid can be used as an effective supplement in promoting health in all age and gender groups[24]. 
Fursultiamine is a nutritional supplement and vitamin B1 derivative, with potential antineoplastic activity. Fursultiamine can be used for vitamin $\mathrm{B}_{1}$ deficiency [15-Selleckchem].

Ergosterol is a sterol and a biological precursor (a provitamin) to vitamin D2 (Selleckchem). In recent years, the ergosterol rich extract presented notable antioxidant and antimicrobial properties, besides showing no hepatotoxicity. In fact, ergosterol is a type of plant sterol found in mushrooms. Mushrooms have been widely consumed as food, especially by the Chinese and Japanese [26].

Riboflavin (Vitamin B2) is a heat-stable and water-soluble vitamin found in food and used as a dietary supplement to prevent and treat riboflavin deficiency. This vitamin is essential for healthy skin, nails, and hair [15 - Selleckchem].

Vitamin B12 is a water soluble vitamin with a key role in the normal functioning of the brain and nervous system (Selleckchem). It is better to use natural resources to meet the body's need for vitamin B12, but doctors sometimes prescribe fortified foods and supplements for people over the age of 50 [29].

\section{Conclusion}

In this study, virtual screening was successfully used to identify FDA 47 approved vitamins, which having therapeutic activity against the viral Mpro enzyme and S-RBD spike protein of COVID-19. It was observed that Dibenzoyl Thiamine, Folic Acid, Riboflavin, Fursultiamine, Benfotiamine, Ergosterol, Cocarboxylase and Calcipotriene were found to be potential inhibitors against the viral Mpro enzyme and Vitamin B12, Folic Acid, Dibenzoyl Thiamine and Benfotiamine for S-RBD spike protein of COVID-19.

\section{Declarations}

Funding: No funding was received for this work.

Conflicts of interest/Competing interests: The authors declare that there is no conflict of interest

Ethics approval: This study does not require ethics approval.

Consent to participate: This study does not require participate approval.

Consent for publication: This study does not require publication approval

Availability of data and material: The datasets used and/or analyzed during the current study are available from the corresponding author on reasonable request.

Code availability: The software used during this study are freely available and are also available from the corresponding author on reasonable request.

Authors' contributions: This manuscript has been done by myself and there was no co-author 


\section{References}

[1] Data obtained from https://covid19.who.int/ (World Health Organization)

[2] Fehr, A.R., Perlman, S. Coronaviruses: an overview of their replication and pathogenesis. Methods Mol Biol. 2015, 1282, 1-23.

[3] Yang, P., Wang, X. COVID-19: a new challenge for human beings. Cell Mol Immunol. 2020, 17, 555-7.

[4] Wu, F., Zhao, S., Yu, B., Chen, Y.M., Wang, W., Song, Z.G., et al. A new coronavirus associated with human respiratory disease in China. Nature. 2020, 579, 265-9.

[5] Chen, Y.W., Yiu, C.B., Wong, K.Y. Prediction of the SARS-CoV-2 (2019-nCoV) 3C-like protease (3CL (pro)) structure: virtual screening reveals velpatasvir, ledipasvir, and other drug repurposing candidates. F1000Res. 2020, 9, 129.

[6] Khan, S.A., Zia, K., Ashraf, S., Uddin, R., Ul-Haq, Z. Identification of chymotrypsin-like protease inhibitors of SARS-CoV-2 via integrated computational approach. J Biomol Struct Dyn. 2020, 1-10.

[7] Gahlawat, A., Kumar, N., Kumar, R., Sandhu, H., Singh, I.P., Singh, S., et al. Structure-Based Virtual Screening to Discover Potential Lead Molecules for the SARS-CoV-2 Main Protease. J Chem Inf Model. 2020.

[8] Schoeman, D., Fielding, B.C. Coronavirus envelope protein: current knowledge. Virol J. 2019, 16, 69.

[9] Ravichandran, S., Coyle, E.M., Klenow, L., Tang, J., Grubbs, G., Liu, S., et al. Antibody signature induced by SARS-CoV-2 spike protein immunogens in rabbits. Sci Transl Med. 2020, 12.

[10] Unni, S., Aouti, S., Thiyagarajan, S., Padmanabhan, B. Identification of a repurposed drug as an inhibitor of Spike protein of human coronavirus SARS-CoV-2 by computational methods. J Biosci. 2020, 45 .

[11] Chikhale, R.V., Gurav, S.S., Patil, R.B., Sinha, S.K., Prasad, S.K., Shakya, A., et al. Sars-cov-2 host entry and replication inhibitors from Indian ginseng: an in-silico approach. J Biomol Struct Dyn. 2020, 1-12.

[12] Berman, H.M., Westbrook, J., Feng, Z., Gilliland, G., Bhat, T.N., Weissig, H., et al. The Protein Data Bank. Nucleic Acids Res. 2000, 28, 235-42.

[13] Visualizer, A.D.S. Version 4.5. Softw. Vis. Anal. Protein Struct. 2017.

[14] Morris, G.M., Huey, R., Lindstrom, W., Sanner, M.F., Belew, R.K., Goodsell, D.S., et al. AutoDock4 and AutoDockTools4: Automated docking with selective receptor flexibility. J Comput Chem. 2009, 30, 278591. 
[15] Bolton, E.E., Wang, Y., Thiessen, P.A., Bryant, S.H. PubChem: integrated platform of small molecules and biological activities. In: Annual reports in computational chemistry, Elsevier, 2008, Vol. 4, pp. 217-41.

[16] Hanwell, M.D., Curtis, D.E., Lonie, D.C., Vandermeersch, T., Zurek, E., Hutchison, G.R. Avogadro: an advanced semantic chemical editor, visualization, and analysis platform. J Cheminform. 2012, 4, 17.

[17] Dallakyan, S., Olson, A.J. Small-molecule library screening by docking with PyRx. Methods Mol Biol. $2015,1263,243-50$.

[18] Laskowski, R.A., Swindells, M.B. LigPlot+: multiple ligand-protein interaction diagrams for drug discovery. J Chem Inf Model. 2011, 51, 2778-86.

[19] Berendsen, H.J., van der Spoel, D., van Drunen, R. GROMACS: a message-passing parallel molecular dynamics implementation. Computer physics communications. 1995, 91, 43-56.

[20] Lindorff-Larsen, K., Piana, S., Palmo, K., Maragakis, P., Klepeis, J.L., Dror, R.O., et al. Improved sidechain torsion potentials for the Amber ff99SB protein force field. Proteins. 2010, 78, 1950-8.

[21] Schrödinger, L. PyMOL. The PyMOL Molecular Graphics System, Version. 2017, 2.

[22] Yoshida, M., Hishiyama, T., Igarashi, T. A novel method for determining total vitamin B1 in processed food enriched with dibenzoyl thiamine. Journal of the Japanese Society for Food Science and Technology (Japan). 2008.

[23] Heywood, R., Wood, J., Majeed, S. Tumorigenic and toxic effect of O, S-dibenzoyl thiamine hydrochloride in prolonged dietary administration to rats. Toxicology letters. 1985, 26, 53-8.

[24] Bae, S., Kamynina, E., Farinola, A.F., Caudill, M.A., Stover, P.J., Cassano, P.A., et al. Provision of folic acid for reducing arsenic toxicity in arsenic-exposed children and adults. The Cochrane Database of Systematic Reviews. 2017, 2017.

[25] Authority, E.F.S. Benfotiamine, thiamine monophosphate chloride and thiamine pyrophosphate chloride, as sources of vitamin B1 added for nutritional purposes to food supplements-Scientific Opinion of the Panel on Food Additives and Nutrient Sources added to Food (ANS). EFSA Journal. 2008, 6, 864.

[26] Corrêa, R.C., Barros, L., Fernandes, Â., Sokovic, M., Bracht, A., Peralta, R.M., et al. A natural food ingredient based on ergosterol: optimization of the extraction from Agaricus blazei, evaluation of bioactive properties and incorporation in yogurts. Food \& function. 2018, 9, 1465-74.

[27] Satake, K., Amano, T., Okamoto, T. Calcipotriol and betamethasone dipropionate synergistically enhances the balance between regulatory and proinflammatory $\mathrm{T}$ cells in a murine psoriasis model. Scientific reports. 2019, 9, 1-11. 
[28] Ben-Eltriki, M., Deb, S., Guns, E.S.T. Calcitriol in combination therapy for prostate cancer: pharmacokinetic and pharmacodynamic interactions. Journal of Cancer. 2016, 7, 391.

[29] Vogel, T., Dali-Youcef, N., Kaltenbach, G., Andres, E. Homocysteine, vitamin B12, folate and cognitive functions: a systematic and critical review of the literature. International journal of clinical practice. 2009, $63,1061-7$.

[30] Naqvi, A.A.T., Fatima, K., Mohammad, T., Fatima, U., Singh, I.K., Singh, A., et al. Insights into SARSCoV-2 genome, structure, evolution, pathogenesis and therapies: Structural genomics approach. Biochimica et Biophysica Acta (BBA)-Molecular Basis of Disease. 2020, 165878.

\section{Tables}


Table 1

Top screened vitamins against Main Protease (section I) and RBD domain (section II) of Spike protein

$\begin{array}{llll}\text { Name } & \text { Pubchem } & \begin{array}{l}\text { Docking } \\ \text { Score } \\ \text { (Kcal/mol) }\end{array} & \text { HP }\end{array}$

HB

Section I

Indinavir

5362440

$-8.1$

T24, T25, T26, H41, M49. F140, L141, E166

N142, H163, H164, M165, P168, R188,

Q189 and T190

Dibenzoyl

$3036235 \quad-7.9$

H41, L141, N142, H164, M165, and

Q189

G143, S144,

C145 and

E166

$\begin{array}{llll}\text { Folic Acid } & \text { 135398658 } & -7.9 & \begin{array}{l}\text { T24, T26, H41, S46, M49, G143, C1 } \\ \text { H164, M165, Q189 }\end{array} \\ \text { Riboflavin } & 493570 & -7.7 & \begin{array}{l}\text { H41, Met49, F140, E166, M165 and } \\ \text { Q189 }\end{array}\end{array}$

Fursultiamine $\quad 3002119 \quad-7.0$

T26, T25, H41, M49, N142, H164,

M165, D187 and Q189

T25, T45

and E166

L141, N142,

G143, S144

and C145

T26, L141,

G143, S144

and C145

$\begin{array}{lcclll}\text { Ergosterol } & 444679 & -7.7 & \begin{array}{l}\text { M49, N142, G143, C145, M165, E166, } \\ \text { R188, Q189 and T190 }\end{array} & \text { T26 } \\ \text { Calcipotriene } & 5288783 & -7.2 & \begin{array}{l}\text { T25, H41, M49, L141, N142, C145, } \\ \text { E166 and Q189 }\end{array} & \begin{array}{l}\text { T26 and } \\ \text { G143 }\end{array}\end{array}$

Cocarboxylase 9068

$-7.1$

H41, L141, N142, G143, S144, M165,

E166, R188 and Q189

Y54, C145,

H164 and

D187

$\begin{array}{lllll}\text { Benfotiamine } & 3032771 & -7.1 & \begin{array}{l}\text { T25, L27, H41, M49, F140, L141, } \\ \text { N142, C145, M165, E166, D184, R188 } \\ \text { and Q189 }\end{array} & \begin{array}{l}\text { Y54 and } \\ \end{array} \\ & & \begin{array}{l}\text { and } \\ \end{array} & \end{array}$

Vitamin B1 $6042 \quad-6.0$

M49, F140, N142, G143, C145, H163, H164, M165, E166 and Q189

H41 and

L141

Section II

\begin{tabular}{|c|c|c|c|c|}
\hline Vitamin B12 & 5311498 & -7.6 & $\begin{array}{l}\text { K417, L455, F456, Tyr473, Tyr489, } \\
\text { Gln493, S494, Y495, Q498 and Y505 }\end{array}$ & $\begin{array}{l}\text { R403, Y449 } \\
\text { and G496 }\end{array}$ \\
\hline Folic Acid & 135398658 & -7.2 & $\begin{array}{l}\text { R403, D405, E406, Gly416, K417, } \\
\text { Leu455, Y495 and G496 }\end{array}$ & $\begin{array}{l}\text { Q409 and } \\
\text { Y453 }\end{array}$ \\
\hline $\begin{array}{l}\text { Dibenzoyl } \\
\text { Thiamine }\end{array}$ & 3036235 & -7.0 & $\begin{array}{l}\text { Y449, Y453, S494, Y495, N501 and } \\
\text { Y505 }\end{array}$ & $\begin{array}{l}\text { R403, G496 } \\
\text { and Q498 }\end{array}$ \\
\hline
\end{tabular}

HP: Hydrophobic Bond and HB: Hydrogen Bond 


\begin{tabular}{|c|c|c|c|c|}
\hline \multirow[t]{2}{*}{ Name } & \multirow{2}{*}{$\begin{array}{l}\text { Pubchem } \\
\text { ID }\end{array}$} & \multirow{2}{*}{$\begin{array}{l}\text { Docking } \\
\text { Score } \\
\text { (Kcal/mol) }\end{array}$} & \multicolumn{2}{|l|}{ Interaction with $3 \mathrm{CLP}$} \\
\hline & & & $\mathrm{HP}$ & HB \\
\hline Benfotiamine & 3032771 & -6.2 & R403, Y495, Q498, N501 and Y505 & $\begin{array}{l}\text { Y449, Q493, } \\
\text { S494 and } \\
\text { G496 }\end{array}$ \\
\hline
\end{tabular}

\section{Figures}
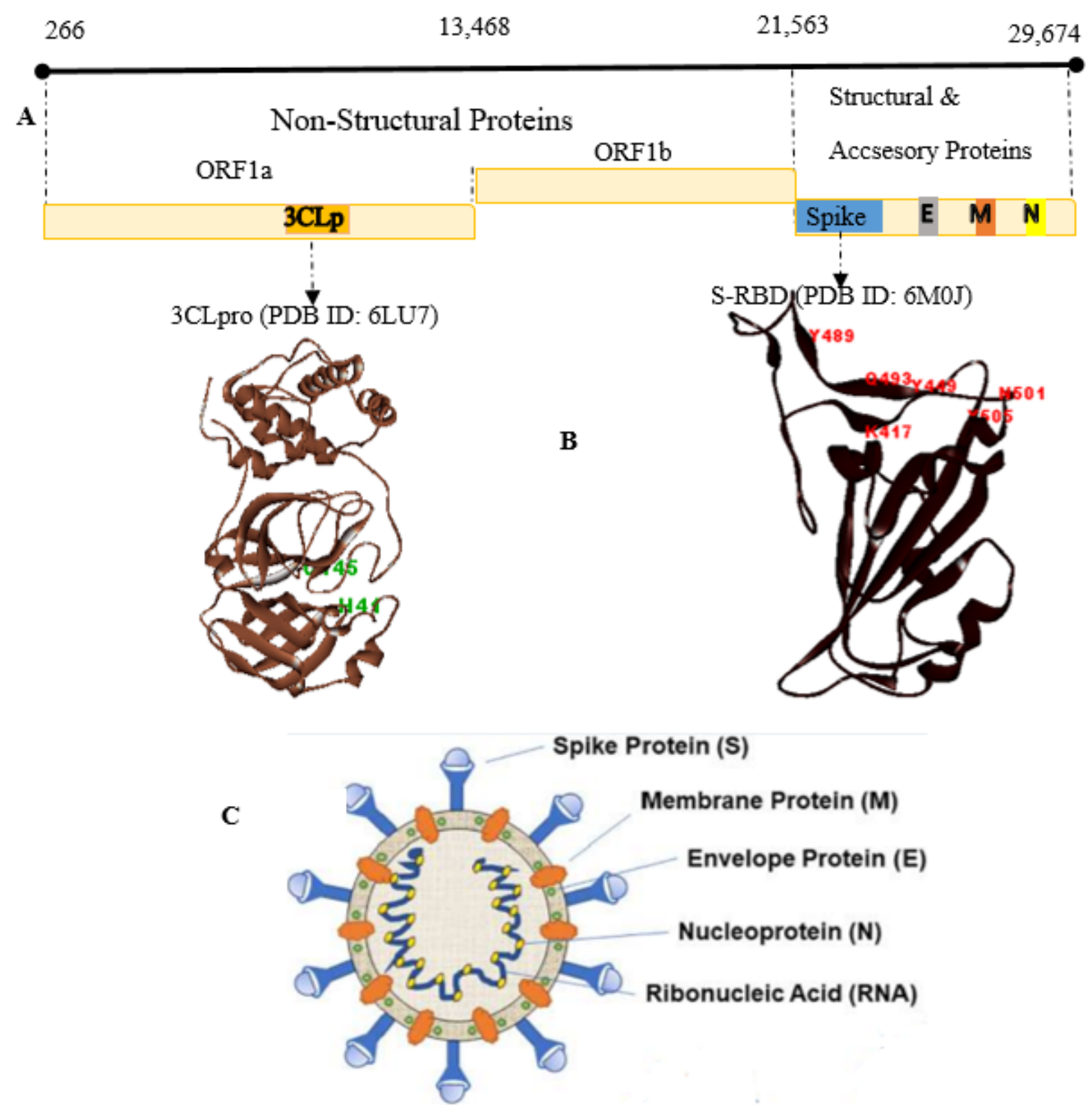

Figure 1 
Schematic representation of the SARS-CoV-2 structure and its genomic organization. A) Schematic representation of genome sequence of SARS-CoV-2 and proteome showing different polyproteins ( $\mathrm{pp} 1 \mathrm{a}$ and $\mathrm{pp} 1 \mathrm{~b}$ ) along with the structural and accessory proteins. B) Structure of 3CLp with catalytic residues and S-RBD which represented the ACE2 binding residues. C) Diagrom of coronavirus structure showing M (membrane) protein, $\mathrm{S}$ (Spike) protein, $\mathrm{E}$ (envelope) protein, $\mathrm{N}$ (nucleocapsid) protein \& RNA along with the ACE2 receptor [30].
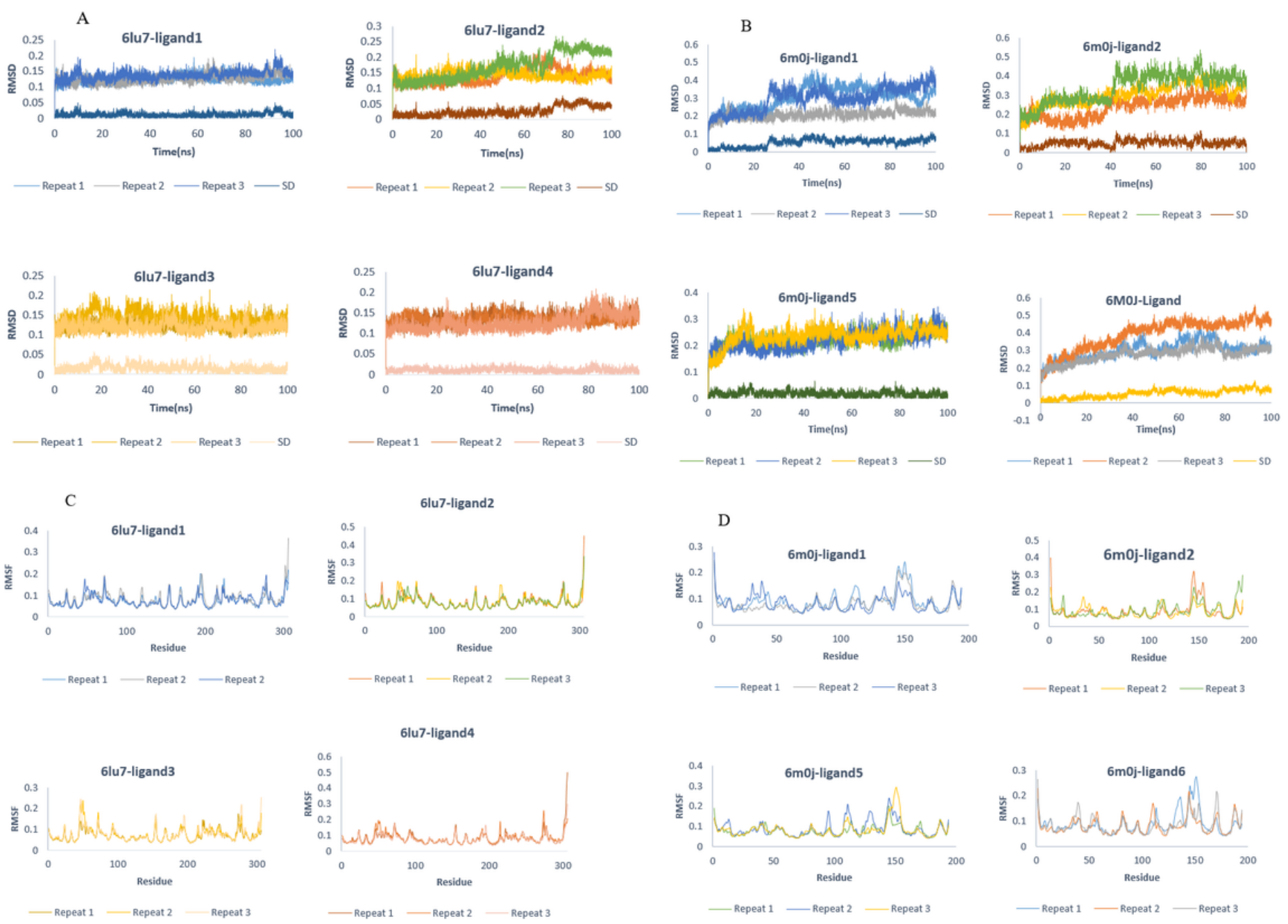

\section{Figure 2}

Analysis of the molecular dynamics simulations for 3CLp-ligand and 6m0j-ligand complexes. A) Root mean square deviation (RMSD) analysis of the protein backbone for the complexes of 6lu7-with Dibenzoyl thiamine (ligand1), Folic Acid (ligand2), Riboflavin (ligand3) and Fursultiamine (ligand4). B) 6m0j-with Dibenzoyl thiamine (ligand_1), folic acid (ligand_2), Benfotiamine (ligand_5) and vitamin B12 (ligand_6). C) Root mean square fluctuation (RMSF) plot for the protein backbone atoms for 3CLp-ligand complexes and D) 6m0j-ligand complexes. 

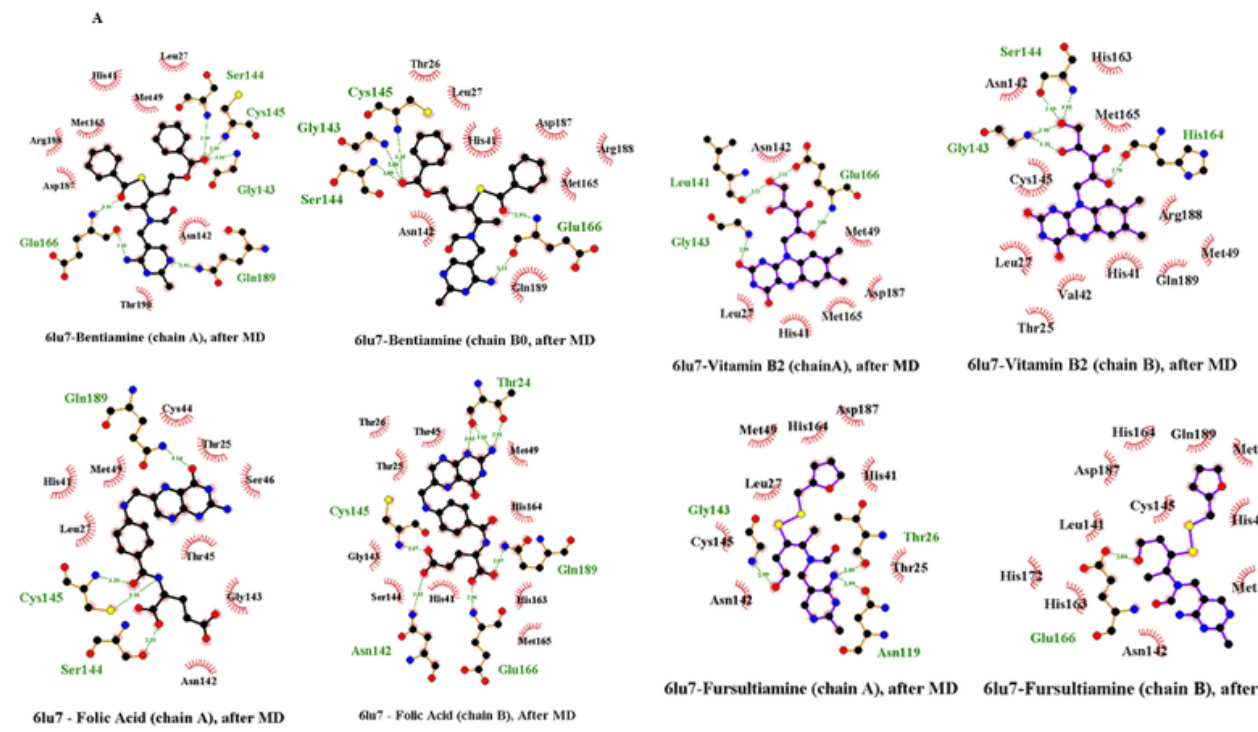

6lu7-Vitamin B2 (chain A), after MD

6lu7-Vitamin B2 (chain B), after MD
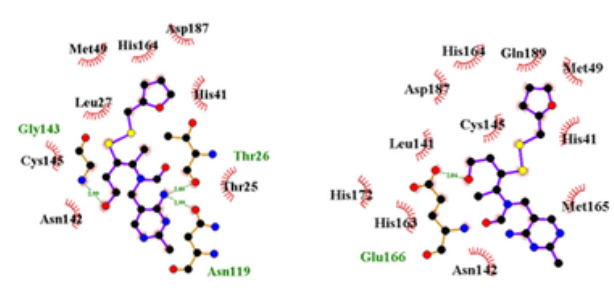

Glu7-Fursultiamine (chain A), after MD 6lu7-Fursultiamine (chain B), after M

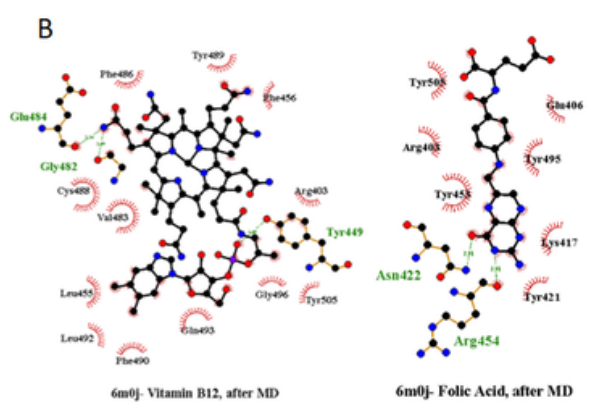

Figure 3

Ligplot analysis of 6lu7-ligands and $6 \mathrm{m0j}$ - ligands interaction. A) 2D representation of the hit compounds in the binding pocket of dimeric form of 33CL- protease (6lu7) after molecular dynamics for 6lu7Bentiamin and 6lu7-folic acid, 6lu7-Vitamin B2 and 6lu7-Fursultiamine. B) 2D representation of the hit compounds in the interface area of S-RBD, after molecular dynamics simulation of $6 \mathrm{m0j}$-Vitamin B12, Folic Acid, Bentiamin and Benfotiamine. Green lines indicate the hydrogen bonds and red dotted lines indicate the hydrophobic interactions.

\section{Supplementary Files}

This is a list of supplementary files associated with this preprint. Click to download.

- supplementaryfigureRevisebeforereview.docx 